\title{
Acute Respiratory Distress Syndrome After Pancreaticoduodenectomy
}

\author{
Takamune Yamaguchi ${ }^{1}$, Takashi Kokudo ${ }^{1}$, Michiro Shiozawa ${ }^{1}$, Nobuhisa Akamatsu' ${ }^{1}$, Junichi Kaneko ${ }^{1}$, \\ Junichi Arita ${ }^{1}$, Yoshihiro Sakamoto ${ }^{1}$, Norihiro Kokudo ${ }^{1,2}$, Kiyoshi Hasegawa ${ }^{1}$
}

Corresponding author:

Kiyoshi Hasegawa,MD

Hepato-Biliary-Pancreatic Surgery

Division, Department of Surgery,

Graduate School of Medicine, The

University of Tokyo, Tokyo, Japan

7-3-1 Hongo, Bunkyo-ku, Tokyo, Japan

Phone: +81338155411

Fax: +81356843989

E-mail: kihase-tky@umin.ac.jp

\section{Abbreviations:}

ARDS - Acute respiratory distress syndrome

PD - pancreaticoduodenectomy

IP - interstitial patterns
Received: 21.09 .2018

Accepted: 15.11 .2018

Copyright () Celsius Publishing House www.sgo-iasgo.com

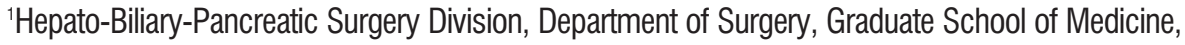
The University of Tokyo, Tokyo, Japan

${ }^{2}$ National Center for Global Health and Medicine, Tokyo, Japan

\section{ABSTRACT}

Introduction: Acute respiratory distress syndrome (ARDS) can cause severe morbidity and mortality after a pancreaticoduodenectomy (PD). However, very few studies have evaluated the risk factors for ARDS among patients undergoing abdominal surgery, with even fewer studies being performed for patients undergoing PD specifically.

Methods: We performed a retrospective chart analysis of 200 patients undergoing PD between February 2009 and April 2014 in our Department and investigated the risk factors for ARDS by analysing preoperative chest CT scan images and other clinical parameters.

Results: Among the 200 patients, interstitial patterns (IP) on preoperative lung CT images were found in 12 patients (6\%, IP group). Of these, ARDS developed in 2 patients (16.7\%), whereas ARDS did not occur in any of the remaining 188 patients without an IP pattern. Both of the ARDS patients died within 90 days without experiencing any other abdominal complications. The serum KL-6 level was measured in 6 of the 12 patients in the IP group, and the KL- 6 levels were higher than the normal limit for our hospital $(500 \mathrm{U} / \mathrm{mL})$ in 2 patients. One of these 2 patients developed ARDS after PD. No other clinical findings or parameters predictive of ARDS after PD were found.

Conclusions: ARDS after PD can occur in patients without any history of respiratory disease and is associated with a very high operative mortality. Interstitial patterns on preoperative lung CT images may predict a risk of ARDS after PD.

Key words: acute respiratory distress syndrome, pancreaticoduodenectomy

\section{INTRODUCTION}

Acute respiratory distress syndrome (ARDS) is a life-threatening condition (1) and a leading cause of mortality in hospital intensive care units (2). Despite advances in understanding of the aetiology and treatment of this disorder, ARDS continues to be associated with a high mortality rate. Among patients admitted to an ICU, 7.1\% develop ARDS (3). The overall prevalence of ARDS is reported to be $2.2 \%$ to $4.2 \%$ among patients who have undergone lung resection; among these patients, the mortality rate from ARDS ranged from $52 \%$ to $65 \%(4,5)$.

Several risk factors for ARDS after partial lung resection have been identified, including age $>60$ years, male gender, chronic lung disease, reduced respiratory 
function, prior radiation or chemotherapy, concurrent cardiac disease, type and extent of lung resection, increased blood loss, blood transfusions, an excessive volume of intraoperative fluids, and reoperation (6-10). A higher $\mathrm{KL}-6$ value $(\geq 1000 \mathrm{U} / \mathrm{mL}$ ) may also indicate a risk of developing ARDS after lung resection(11). However, the risk factors for ARDS after abdominal surgery remain unknown.

Only a few reports on ARDS after abdominal surgery have been published. Haga et al reported that the prevalence of ARDS was $1.3 \%$ after gastrointestinal surgery, including esophagectomy, pancreaticoduodenectomy (PD), hepatectomy, gastrectomy, colorectal resection, and laparoscopic cholecystectomy; the subsequent mortality rate, however, was not mentioned (12). Brenner et al reported a case of ARDS after a metastatic liver resection (13). However, no report focusing on ARDS after pancreatectomy has been published to date.

The cause of ARDS also remains unknown, and no effective treatments have been established. Although the incidence of ARDS after PD may be relatively low, once ARDS occurs, it adds an additional life-threatening risk to one of the most invasive abdominal operations presently performed.

In this report, we investigated the incidence of subclinical interstitial pneumonia among patients undergoing PD and the risk factors for postoperative ARDS. We also describe the clinical courses of 2 cases with ARDS after PD.

\section{MATERIALS AND METHODS}

\section{Patient characteristics}

A total of 200 patients who underwent PD between February 2009 and April 2014 at a tertiary care hospital in Japan were retrospectively analysed. The demographic data of the patients and the histopathological features are summarized in table 1 . We analysed the surgical duration, blood loss, and postoperative morbidity and mortality rates. Operative mortality was defined as death within 90 days after surgery or before hospital discharge. Postoperative

Table 1 - Patient demographics and histopathological features

\begin{tabular}{|c|c|c|c|c|}
\hline & IP group & No IP group & Overall & $P$ value \\
\hline \multicolumn{5}{|l|}{ Patient demographics } \\
\hline Number & 12 & 188 & 200 & \\
\hline Age in years, median (range) & $76.5(59-82)$ & $69.5(35-84)$ & $70(35-84)$ & 0.0033 \\
\hline Sex & & & & 0.24 \\
\hline Male, $\mathrm{n}(\%)$ & $10(83.3)$ & $126(67.0)$ & $136(68)$ & \\
\hline Female, $\mathrm{n}(\%)$ & $2(16.7)$ & $62(33.0)$ & $64(32)$ & \\
\hline BMI, median (range) & $23.3(15.9-28.1)$ & $21.9(13.7-32.5)$ & $22.0(13.7-32.5)$ & 0.24 \\
\hline ASA classification & & & & 0.45 \\
\hline $\mathrm{I}, \mathrm{n}(\%)$ & $2(16.7)$ & $71(37.8)$ & $73(36.5)$ & \\
\hline $\mathrm{II}, \mathrm{n}(\%)$ & $9(75.0)$ & $109(58.0)$ & $118(59.0)$ & \\
\hline III/IV, n (\%) & $1(8.3)$ & $8(4.2)$ & $9(4.5)$ & \\
\hline Brinkman index & $480(0-2400)$ & $300(0-6000)$ & $310(0-6000)$ & 0.20 \\
\hline SmokingYes, n (\%)/No, n (\%) & $10(83.3) / 2(16.7)$ & $116(61.7) / 72(38.3)$ & $126(63) / 74(37)$ & 0.13 \\
\hline Type of indication & & & & 0.74 \\
\hline Pancreatic adenocarcinoma, n (\%) & $6(50.0)$ & $76(40.4)$ & $82(41)$ & \\
\hline Distal common bile duct adenocarcinoma, $n(\%)$ & $3(25)$ & $28(14.9)$ & $31(15.5)$ & \\
\hline Ampullary adenocarcinoma, $\mathrm{n}(\%)$ & $1(8.3)$ & $25(13.3)$ & $26(13)$ & \\
\hline Duodenal adenocarcinoma, $\mathrm{n}(\%)$ & $0(0)$ & $13(6.9)$ & $13(6.5)$ & \\
\hline Other malignant disease, $\mathrm{n}(\%)$ & $1(8.3)$ & $6(3.2)$ & $7(3.5)$ & \\
\hline Neuroendocrine tumor, $\mathrm{n}(\%)$ & $1(8.3)$ & $9(4.8)$ & $10(5)$ & \\
\hline Intraductal papillary-mucinous neoplasms, n (\%) & $0(0)$ & $29(15.4)$ & $29(14.5)$ & \\
\hline Others, $\mathrm{n}(\%)$ & $0(0)$ & $2(1)$ & $2(1)$ & \\
\hline
\end{tabular}

BMI: body mass index, ASA classification: the American Society of Anesthesiologists classification, IP group: group with an interstitial pattern visible on preoperative CT images 
complications were rated according to the ClavienDindo classification (14). Pancreaticojejunostomy leakage and post-pancreatectomy haemorrhage were each analysed according to the consensus definitions by the International Study Group of Pancreatic Surgery (ISGPS) $(15,16)$. Patients with grades B or C were treated as being positive for complications.

\section{Acute respiratory distress syndrome (ARDS)}

Among the 200 patients, two patients developed postoperative ARDS (1\%). The perioperative management protocol has been described elsewhere (17). In addition to a history and physical examination, preoperative evaluations including a chest radiography, pulmonary function tests, electrocardiography (ECG), and computerized tomography (CT) scans of the chest and abdomen were performed. Echocardiography (UCG), and positron emission tomography (PET) or brain imaging were performed to evaluate the disease extent, when necessary. PD was performed using a modified Child reconstruction. In cases with a narrow main pancreatic duct and/or pancreas with a soft texture, we performed a 2-stage pancreatojejunostomy (17).

ARDS was defined as the acute onset of hypoxemia with abnormal oxygenation ratios [arterial oxygen pressure $\left(\mathrm{PaO}_{2}\right) /$ fraction of inspired oxygen ( $\mathrm{FIO} 2$ ) less than 200] and radiographic infiltration characteristics suggestive of pulmonary oedema according to the American-European Consensus Conference on ARDS guidelines (6).

If respiratory failure (ARDS) occurred during the postoperative period, the patients were transferred to the ICU, where an arterial blood gas analysis and chest radiography were performed upon admission and daily thereafter. Patients were ventilated using low-tidalvolume ventilation and positive end expiratory pressure levels. Steroids, sivelestat, and antibiotics were also administered.

\section{Risk factors for ARDS}

The possible risk factors that were investigated consisted of an interstitial pattern on preoperative CT images, the serum KL-6 level, the Brinkman index (18), and the body mass index. All the interstitial patterns were diagnosed by radiologists.

\section{Endpoints}

The main outcomes were the analysed preoperative chest CT images, the serum KL- 6 level, and postoperative ARDS. Secondary outcome measures were the surgical duration, blood loss, and the postoperative morbidity and mortality rates. Comparisons were performed between 2 groups: an IP group [interstitial pattern (IP) on preoperative CT scan images] ( $n=12$, $6 \%)$, and a non-IP ( $n=188,94 \%)$ group.

\section{Statistical analysis}

The statistical analysis was performed using JMP 13 software (SAS Institute Inc., Cary, NC). Categorical variables were analysed using the chi-squared test or the Fisher exact test, as appropriate. Continuous variables were analyzed using the Student t-test or the Mann-Whitney $U$ test, as appropriate. A P value less than 0.05 was considered to indicate statistical significance.

\section{RESULTS}

\section{Patients' characteristics}

Two hundred patients who underwent treatment between February 2009 and April 2014 were included in the study. Overall, 136 (68\%) patients were male and $64(32 \%)$ were female, with a median age of 70 years (range: 35-84 years). The pathological diagnoses responsible for the surgical indications were pancreatic adenocarcinoma (41\%), distal common bile duct adenocarcinoma (15.5\%), ampullary adenocarcinoma (13\%), and duodenal adenocarcinoma (6.5\%) (table 1). The patients' demographics, body mass indices, American Society of Anesthesiologists (ASA) classifications, Brinkman indices, smoking habits, and indications for surgery were similar between the 2 groups, but the patients' ages weres ignificantly different (median, 76.5 years vs. 69.5 years, $\mathrm{P}=0.0033$ ).

\section{Abnormal findings on thoracic CT images obtained before surgery}

Among the 200 patients who underwent chest CT examinations prior to PD, an interstitial pneumonia pattern (IP) was observed by radiologists in 12 patients (6\%). Other abnormal findings are summarized in table 2.

\section{Postoperative outcomes}

Table 3 shows the postoperative outcomes. The median operative time was 589.5 minutes (range: 254- 
Table 2 - Abnormal findings on thoracic CT images before surgery

\begin{tabular}{|c|c|c|c|c|}
\hline & IP group & No IP group & Overall & P value \\
\hline Number & 12 & 188 & 200 & \\
\hline Intestinal pneumonia pattern, n (\%) & $12(100)$ & $0(0)$ & $12(6)$ & - \\
\hline Chronic obstructive pulmonary disease, $\mathrm{n}(\%)$ & $0(0)$ & 13 & $13(6.5)$ & 0.34 \\
\hline Pleural wall thickning, $\mathrm{n}(\%)$ & $0(0)$ & $5(2.7)$ & $5(2.5)$ & 0.56 \\
\hline Benign nodules, $\mathrm{n}(\%)$ & $2(16.7)$ & $42(22.3)$ & $44(22)$ & 0.64 \\
\hline \multicolumn{5}{|l|}{ Old tuberculosis, n (\%) } \\
\hline Old tuberculosis & $0(0)$ & $4(2.1)$ & $4(2)$ & 0.60 \\
\hline
\end{tabular}

IP group: group with an interstitial pattern visible on preoperative CT images

Table 3 - Surgical outcomes of patients who underwent PD

\begin{tabular}{|c|c|c|c|c|}
\hline & IP group & No IP group & Overall & P value \\
\hline \multicolumn{5}{|l|}{ Surgical outcomes } \\
\hline Surgical duration in minutes, median (range) & $644.5(430-806)$ & $588(254-1148)$ & $589.5(254-1148)$ & 0.14 \\
\hline Blood loss in $\mathrm{mL}$, median (range) & $690(180-2750)$ & $665(50-4350)$ & $670(50-4350)$ & 0.37 \\
\hline \multicolumn{5}{|l|}{ Morbidity } \\
\hline Clavien-Dindo classification & & & & 0.001 \\
\hline Grade 1 & 2 & 21 & 23 & \\
\hline Grade 2 & 2 & 85 & 87 & \\
\hline Grade 3a & 2 & 39 & 41 & \\
\hline Grade $3 b$ & 0 & 8 & 8 & \\
\hline Grade 4a & 0 & 1 & 1 & \\
\hline Grade $4 b$ & 0 & 0 & 0 & \\
\hline Grade 5 & 2 & 1 & 3 & \\
\hline$>$ Grade $3 a, n(\%)$ & $4(33.3)$ & $49(26.0)$ & $53(26.5)$ & 0.58 \\
\hline \multicolumn{5}{|l|}{ Morbidity (>Grade 3a and/or ISGPS > Grade B) } \\
\hline Pancreatojejunostomy leakage, n (\%) & $3(25.0)$ & $52(27.7)$ & $55(27.5)$ & 0.84 \\
\hline Post-pancreatectomy hemorrhage, n (\%) & $1(8.3)$ & $8(4.3)$ & $9(4.5)$ & 0.50 \\
\hline Hepaticojejunostomy leakage, $n(\%)$ & $0(0)$ & $4(2.1)$ & $4(2)$ & 0.60 \\
\hline Primary intra-abdominal abcess, $n(\%)$ & $0(0)$ & $5(2.7)$ & $5(2.5)$ & 0.56 \\
\hline \multicolumn{5}{|l|}{ Respratory complications } \\
\hline ARDS, $n(\%)$ & $2(16.7)$ & 0 & $2(1)$ & $<.0001$ \\
\hline Pneumonia, $\mathrm{n}(\%)$ & $2(16.7)$ & $9(4.8)$ & $11(5.5)$ & 0.08 \\
\hline Pneumothorax, n (\%) & $0(0)$ & $1(0.5)$ & $1(0.5)$ & 0.80 \\
\hline
\end{tabular}

IP group: group with an interstitial pattern visible on preoperative CT images , ARDS: acute respiratory distress syndrome

1148 minutes), and the median estimated blood loss was $670 \mathrm{~mL}$ (range: $50-4350 \mathrm{~mL}$ ). Major complications (Clavien-Dindo classification > Grade $3 a$ or ISGPS > Grade B) occurred in 53 patients (26.5\%): pancreaticojejunostomy leakage occurred in 55 patients (27.5\%), post-pancreatectomy haemorrhage occurred in 9 patients (4.5\%), hepaticojejunostomy leakage occurred in 4 patients (2\%), and an intra-abdominal abscess occurred in 5 patients (2.5\%). The 90-day post-operative mortality rate was $1.5 \%$ (3 patients). There were no significant differences in the surgical duration, blood loss, mortality rates, or rates of pancreaticojejunostomy leakage, post-pancreatectomy haemorrhage, hepaticojejunostomy leakage or intra-abdominal abscess between the IP and non-IP groups (table 3).

\section{Respiratory complications and KL-6 levels}

Two $(16.7 \%)$ of the 12 patients in the IP group developed ARDS ( $P=0.0033$; table 3, fig. 1), whereas 


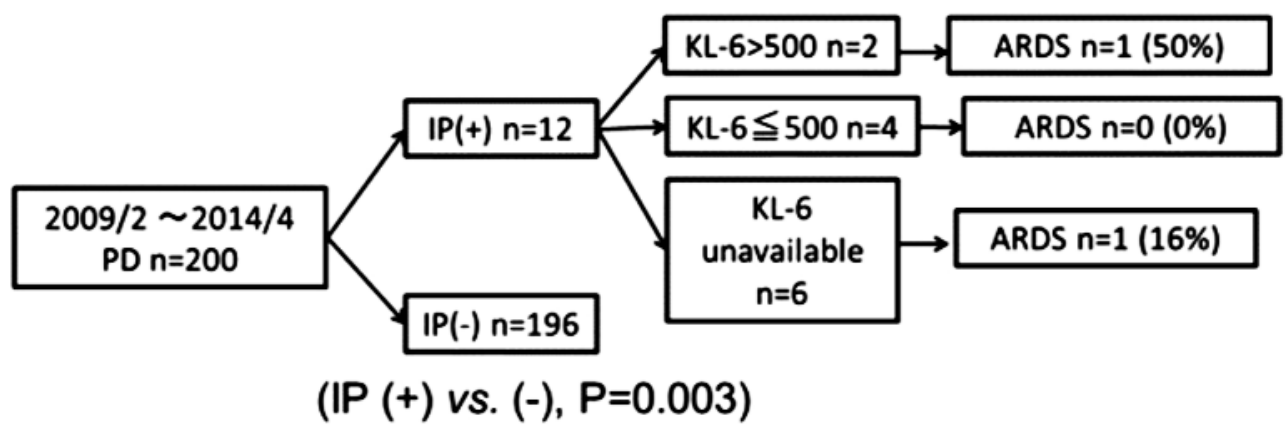

Figure 1 - Acomparison between the IP and non-IP group shows higher rates of ARDS in the IP group (16.7\%vs. $0 \%, P=0.003)$. The serum KL- 6 levelwas preoperatively measured in 6 out of 12 patients and was found to be higher than the upper limit of our hospital $(500 \mathrm{U} / \mathrm{mL})$ in 2 patients, one of who developed ARDS after PD.

*PD: pancreatoduodenectomy; **IP: interstitial patterns in the preoperative chest CT (as diagnosed by a radiologist)

none of the 188 patients in the non-IP group developed ARDS (0\%). Other respiratory complications were pneumonia ( 2 patients vs. 9 patients, $\mathrm{P}=0.08$ ) and pneumothorax (0 patient vs. 1 patient, $P=0.80$ ) (table 2).

The serum KL-6 levels were analysed in 6 of the 200 patients, among whom the KL-6 levels were higher than the normal range $(>500 \mathrm{U} / \mathrm{mL})$ in 2 patients $(770$ and $714 \mathrm{U} / \mathrm{mL}$ ). Among the remaining 4 patients with normal KL-6 levels (207, 396, 390 and $489 \mathrm{U} / \mathrm{mL}$, respectively), ARDS developed in 1 patient after PD. In the other patient who developed ARDS, the KL- 6 level was not measured (fig. 1).

Case 1. A 79-year old female patient with a medical history of hypertension presented with symptoms of jaundice and lack of appetite. An abdominal CT scan revealed a tumour near the lower part of the common bile duct. A 2-cm diameter nodule in the right lower part of the lung and interstitial patterns in both lower lung fields (fig. 2: left side) were observed on a chest CT scan. A partial lung resection was performed to exclude pulmonary metastases. However, the pathological diagnosis was mucosa-associated lymphoid tissue (MALT) lymphoma. She underwent a PD 14 days after the lung resection. The operative procedure was uneventful, bleeding was minimal $(400 \mathrm{~mL})$, no transfusion was necessary, and no intraoperative respiratory complications arising from the general anaesthesia occurred. On postoperative day 4 she presented with dyspnoea, hypoxemia, and a consciousness disorder. A CT scan revealed pulmonary oedema and consolidations on both lungs (fig. 2: right side). An arterial blood gas analysis revealed severe hypoxemia $\left(\mathrm{PaO}_{2} / \mathrm{FiO}_{2}\right.$ ratio $=150)$, suggesting ARDS. Intubation was performed, and the patient was placed in the intensive care unit. However, her condition worsened even after steroid pulse therapy was repeated 3 times. Finally, she developed a pneumothorax as a result of barotrauma caused by mechanical intubation and died on day 62 .

Case 2. A 73-year-old man with a sudden worsening

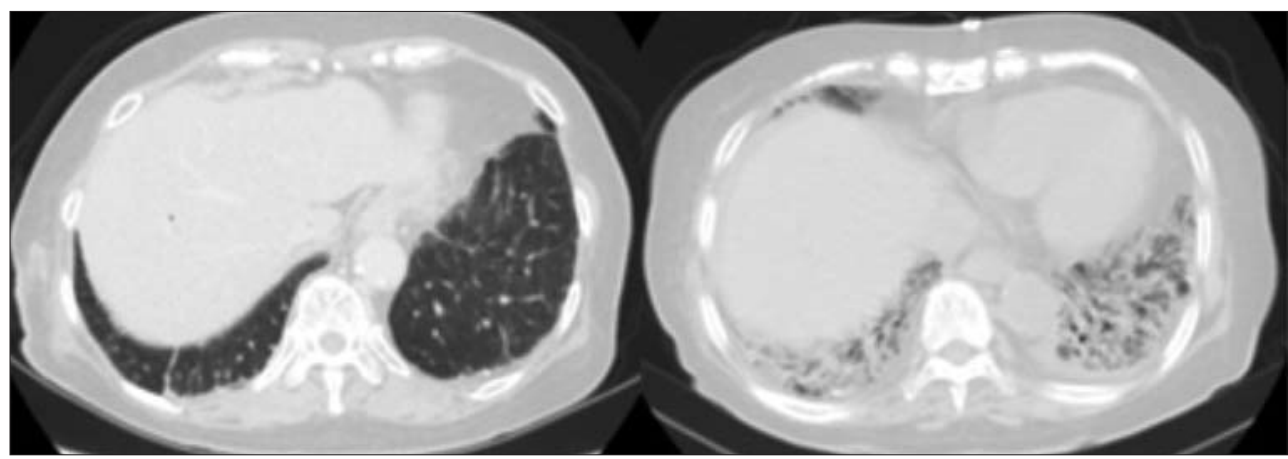

Figure 2 - Patient 1. Interstitial patterns on chest CT images were observed before surgery. The scan obtained on day 4 shows filtration characteristics of pulmonary edema in both lungs. 
of diabetes mellitus, underwent an abdominal CT scan, revealing a tumour in the head of the pancreas. A chest CT showed interstitial patterns (fig. 3: left side). PD was successfully performed with a blood loss of $1400 \mathrm{~mL}$ and without the need for blood transfusion. No intraoperative respiratory complications arising from the general anaesthesia occurred. On postoperative day 3 , he presented with hypoxemia, and a chest X-ray showed infiltrative shadows in both lower lung fields. We suspected pneumonia or mild heart failure, and antibiotics and diuretics were administered. However, his condition worsened. A chest CT scan performed 7 days after the operation revealed pulmonary oedema in both lungs (fig. 3: right side). An arterial blood gas analysis revealed severe hypoxemia $\left(\mathrm{PaO}_{2} / \mathrm{FiO}_{2}\right.$ ratio $=$ 105); thus, we diagnosed the patient as having ARDS. Intubation was performed and the patient was placed in an intensive care unit, but his condition did not improve with medication. Mechanical intubation was necessary beginning on postoperative day 17 , and we repeated steroid pulse therapy twice. Finally, he developed pneumothorax as a result of barotrauma caused by the mechanical intubation, and his condition gradually worsened until he died on postoperative day 26.

\section{DISCUSSION}

We experienced 2 fatal cases of postoperative ARDS after PD. Despite intensive care, both patients died within 90 days after surgery. Although the number of patients in this series is limited, an interstitial pattern on preoperative CT images and a high $\mathrm{KL}-6$ value of more than $500 \mathrm{U} / \mathrm{mL}$ may be risk factors for ARDS after PD.

ARDS develops as a result of an inflammatory process that occurs at the alveolar-capillary interface in the lungs, where the blood in the capillaries remains separate from the gas present in the alveoli (19). According to the American-European Consensus Conference (AECC) on ARDS guidelines (6), acute lung injury and ARDS are defined as the acute onset of hypoxemia with abnormal oxygenation ratios and radiographic infiltration characteristics of pulmonary oedema. Specifically, ARDS was defined as an arterial partial pressure of oxygen relative to the fraction of inspired oxygen $\left[\mathrm{PaO}_{2} / \mathrm{FiO}_{2}\right]$ of $\leq 200 \mathrm{mmHg}$, with no evidence of left atrial hypertension. For a more accurate diagnosis of ARDS, the Berlin definition was developed in 2012 as an initiative of the European Society of Intensive Care Medicine. The Berlin definition of ARDS requires that all four criteria (timing, chest image, origin of edema, and oxygenation) be present for a diagnosis of ARDS. The Berlin definition also recommends three categories of ARDS based on the degree of hypoxemia: mild $\left(200 \mathrm{~mm} \mathrm{Hg}<\mathrm{PaO}_{2} / \mathrm{FiO}_{2}\right.$ $\leq 300 \mathrm{~mm} \mathrm{Hg})$, moderate $\left(100 \mathrm{~mm} \mathrm{Hg}<\mathrm{PaO}_{2} / \mathrm{FiO}_{2} \leq\right.$ $200 \mathrm{~mm} \mathrm{Hg})$, and severe $\left(\mathrm{PaO}_{2} / \mathrm{FiO}_{2} \leq 100 \mathrm{~mm} \mathrm{Hg}\right)$, with a PEEP $\geq 5 \mathrm{~cm} \mathrm{H}_{2} \mathrm{O}$. The Berlin definition enables $\mathrm{a}$ better prediction of mortality than the AECC definition, with increasing ARDS stages associated with higher mortality rates (20). Patients with ARDS frequently develop multiple organ failure, including hepatic, renal and hematopoietic failure, and laboratory testing is needed (21).

Currently, there are no established effective treatments to prevent or control ARDS (22-24). However, several therapies are currently being used for ARDS. ARDS is an acute lung inflammatory disease with the involvement of diverse inflammatory cells and mediators. However, the use of anti-inflammatory

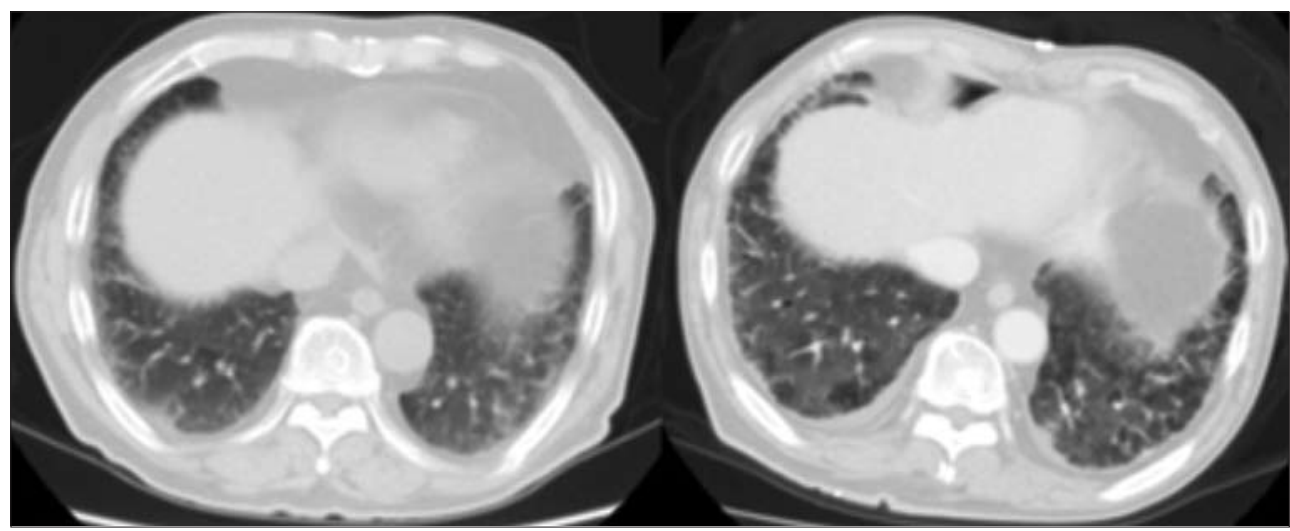

Figure 3 - Patient 2. Interstitial patterns on chest CT images were observed before surgery. The scan obtained on day 7 shows filtration characteristics of pulmonary edema in both lungs. 
corticosteroids does not reportedly improve survival $(21,25)$. The use of neuromuscular blocking agents (NMBAs) as a treatment for ARDS remains controversial. NMBAs might only be beneficial for reducing barotrauma and ventilator-induced lung injury $(26,27)$. According to Tsuboko et al., sivelestat is effective for patients developing ARDS after surgery for abdominal sepsis, enabling early improvements in oxygenation and multiple organ dysfunction (28). Mechanical ventilation with a lower tidal volume $(4-6 \mathrm{~mL} / \mathrm{kg})$ and airway pressure (maintenance of a plateau pressure between 25 and $30 \mathrm{~cm} \mathrm{H} \mathrm{H}_{2} \mathrm{O}$ ) has been reported to reduce the mortality rate among patients with ARDS (29). Prone ventilation has also been reported to be effective in obese patients with ARDS (30).

We attempted all of the possible treatment options for the 2 ARDS patients, both patients died during hospitalization. Our ARDS prevalence rate of $1 \%$ was almost the same as the rates reported in two studies describing patients undergoing lung resection who subsequently developed ARDS.

The risk factors for ARDS after abdominal surgery have not been specifically reported. However, the present findings suggest that an interstitial pattern on preoperative CT images might be a risk factor for ARDS after PD. Furthermore, we had one patient who did not develop any complications after a partial lung resection but who developed ARDS after a PD. A prolonged operation time and major postoperative abdominal complications are risk factors for pulmonary embolization (31). Since PD is one of the most invasive abdominal surgeries presently performed, the development of ARDS may contribute an additional fatal risk.

The relatively small number of cases of ARDS $(n=2)$ among the patients who underwent PD $(n=200)$ at our institution and the investigation of only two independent risk factors were limitations of our study. Further research is required to understand the role of interstitial patterns on preoperative $\mathrm{CT}$ scan images and the preoperative serum KL-6 level in critically ill patients with ARDS after PD.

\section{CONCLUSION}

In conclusion, the presently reported data suggests the importance of preoperative evaluations of interstitial patterns on preoperative CT images, given the potential risk for ARDS after PD. Although further investigation is needed, KL- 6 may be a useful marker for stratifying the risk among patients with radiological IP.

\section{Disclosure}

No conflict of interest or financial support.

\section{REFERENCES}

1. Brodie D, Bacchetta M. Extracorporeal membrane oxygenation for ARDS in adults. N Engl J Med 2011;365:1905-1914.

2. York NL, Kane C. Trends in caring for adult respiratory distress syndrome patients. Dimens Crit Care Nurs 2012;31:153-158.

3. Ferri F. Acute respiratory disress syndrome. Elsevier/Mosby, Philadelphia 2014:57-59.

4. Kutlu CA, Williams EA, Evans TW, Pastorino U, Goldstraw P. Acute lung injury and acute respiratory distress syndrome after pulmonary resection. Ann Thorac Surg 2000;69:376-380.

5. Ruffini E, Parola A, Papalia E, Filosso PL, Mancuso M, Oliaro A, Actis-Dato $\mathrm{G}$, et al. Frequency and mortality of acute lung injury and acute respiratory distress syndrome after pulmonary resection for bronchogenic carcinoma. Eur J Cardiothorac Surg 2001;20:30-36, discussion 36-37.

6. Bernard GR, Artigas A, Brigham KL, Carlet J, Falke K, Hudson L, Lamy $M$, et al. The American-European Consensus Conference on ARDS. Definitions, mechanisms, relevant outcomes, and clinical trial coordination. Am J Respir Crit Care Med 1994;149:818-824.

7. Hayes JP, Williams EA, Goldstraw P, Evans TW. Lung injury in patients following thoracotomy. Thorax 1995;50:990-991.

8. Grichnik KP, D'Amico TA. Acute lung injury and acute respiratory distress syndrome after pulmonary resection. Semin Cardiothorac Vasc Anesth 2004;8:317-334.

9. Dulu A, Pastores SM, Park B, Riedel E, Rusch V, Halpern NA. Prevalence and mortality of acute lung injury and ARDS after lung resection. Chest 2006;130:73-78.

10. Jeon K, Yoon JW, Suh GY, Kim J, Kim K, Yang M, Kim H, et al. Risk factors for post-pneumonectomy acute lung injury/acute respiratory distress syndrome in primary lung cancer patients. Anaesth Intensive Care 2009;37:14-19.

11. Sato T, Teramukai S, Kondo H, Watanabe A, Ebina M, Kishi K, Fujii Y, et al. Impact and predictors of acute exacerbation of interstitial lung diseases after pulmonary resection for lung cancer. J Thorac Cardiovasc Surg 2014;147:1604-1611 e1603.

12. Haga Y, Beppu T, Doi K, Nozawa F, Mugita N, Ikei S, Ogawa M. Systemic inflammatory response syndrome and organ dysfunction following gastrointestinal surgery. Crit Care Med 1997;25:19942000.

13. Brenner T, Motsch J, Werner J, Grenacher L, Martin E, Hofer S. Rapid-Onset Acute Respiratory Distress Syndrome (ARDS) in a Patient Undergoing Metastatic Liver Resection: A Case Report and Review of the Literature. Anesthesiol Res Pract 2010;2010.

14. Dindo D, Demartines N, Clavien P-A. Classification of Surgical Complications. Annals of Surgery 2004;240:205-213.

15. Bassi C, Dervenis C, Butturini G, Fingerhut A, Yeo C, Izbicki J, Neoptolemos J, et al. Postoperative pancreatic fistula: an international study group (ISGPF) definition. Surgery 2005;138:8-13.

16. Grutzmann R, Ruckert F, Hippe-Davies N, Distler M, Saeger HD. Evaluation of the International Study Group of Pancreatic Surgery definition of post-pancreatectomy hemorrhage in a high-volume center. Surgery 2012;151:612-620.

17. Hasegawa K, Kokudo N, Sano K, Seyama Y, Aoki T, Ikeda M, Hashimoto T, et al. Two-stage pancreatojejunostomy in pancreaticoduodenectomy: a retrospective analysis of short-term results. Am J Surg 2008;196:3-10.

18. Brinkman GL, Coates EO, Jr. The effect of bronchitis, smoking, and occupation on ventilation. Am Rev Respir Dis 1963;87:684-693.

19. Hashiba E, Kimura F, Suzuki Y, Asano T, Ono T, Okawa H, Tsubo T, et al. Combination of extracorporeal membrane oxygenation and highfrequency oscillatory ventilation saved a child with severe ARDS 
after pulmonary resection. J Anesth 2011;25:580-584.

20. Force ADT, Ranieri VM, Rubenfeld GD, Thompson BT, Ferguson ND, Caldwell E, Fan E, et al. Acute respiratory distress syndrome: the Berlin Definition. JAMA 2012;307:2526-2533.

21. Rawal G, Yadav S, Kumar R. Acute Respiratory Distress Syndrome: An Update and Review. J Transl Int Med 2018;6:74-77.

22. Ketoconazole for early treatment of acute lung injury and acute respiratory distress syndrome: a randomized controlled trial. The ARDS Network. JAMA 2000;283:1995-2002.

23. Randomized, placebo-controlled trial of lisofylline for early treatment of acute lung injury and acute respiratory distress syndrome. Crit Care Med 2002;30:1-6.

24. Spragg RG, Lewis JF, Walmrath HD, Johannigman J, Bellingan G, Laterre PF, Witte MC, et al. Effect of recombinant surfactant protein $\mathrm{C}$-based surfactant on the acute respiratory distress syndrome. N Engl J Med 2004;351:884-892.

25. Ruan SY, Lin HH, Huang CT, Kuo PH, Wu HD, Yu CJ. Exploring the heterogeneity of effects of corticosteroids on acute respiratory distress syndrome: a systematic review and meta-analysis. Crit Care 2014;18:R63.

26. Alhazzani W, Alshahrani M, Jaeschke R, Forel JM, Papazian L, Sevransky J, Meade MO. Neuromuscular blocking agents in acute respiratory distress syndrome: a systematic review and meta-analysis of randomized controlled trials. Crit Care 2013; $17: R 43$.

27. O'Gara B, Fan E, Talmor DS. Controversies in the Management of Severe ARDS: Optimal Ventilator Management and Use of Rescue Therapies. Semin Respir Crit Care Med 2015;36:823-834.

28. Tsuboko Y, Takeda S, Mii S, Nakazato K, Tanaka K, Uchida E, Sakamoto A. Clinical evaluation of sivelestat for acute lung injury/ acute respiratory distress syndrome following surgery for abdominal sepsis. Drug Des Devel Ther 2012;6:273-278.

29. Acute Respiratory Distress Syndrome N, Brower RG, Matthay MA, Morris A, Schoenfeld D, Thompson BT, Wheeler A. Ventilation with lower tidal volumes as compared with traditional tidal volumes for acute lung injury and the acute respiratory distress syndrome. N Engl J Med 2000;342:1301-1308.

30. De Jong A, Molinari N, Sebbane M, Prades A, Futier E, Jung B, Chanques $\mathrm{G}$, et al. Feasibility and effectiveness of prone position in morbidly obese patients with ARDS: a case-control clinical study. Chest 2013;143:1554-1561.

31. Kokudo T, Uldry E, Demartines N, Halkic N. Pulmonary embolism: specific risk factor after pancreas resection? Pancreas 2014; 43:891-894. 